\title{
LA-UR-16-26726
}

Approved for public release; distribution is unlimited.

Title: Comprehensive numerical methodology for direct numerical simulations of compressible Rayleigh-Taylor instability

Author(s): $\quad$ Reckinger, Scott James

Livescu, Daniel

Vasilyev, Oleg V

Intended for: Web

Issued: 
Disclaimer:

Los Alamos National Laboratory, an affirmative action/equal opportunity employer, is operated by Triad National Security, LLC for the National Nuclear Security Administration of U.S. Department of Energy under contract 89233218CNA000001. By approving this article, the publisher recognizes that the U.S. Government retains nonexclusive, royalty-free license to publish or reproduce the published form of this contribution, or to allow others to do so, for U.S. Government purposes. Los Alamos National Laboratory requests that the publisher identify this article as work performed under the auspices of the U.S. Department of Energy. Los Alamos National Laboratory strongly supports academic freedom and a researcher's right to publish; as an institution, however, the Laboratory does not endorse the viewpoint of a publication or guarantee its technical correctness. 


\section{Computational Physics and Methods}

\section{Comprehensive numerical methodology for direct numerical simulations of compressible Rayleigh-Taylor instability}
S. Reckinger, scott.reckinger@montana.edu
D. Livescu, livescu@lanl.gov
O. Vasilyev, oleg.vasilyev@colorado.edu

A comprehensive numerical methodology has been developed that handles the challenges introduced by considering the compressive nature of Rayleigh-Taylor instability (RTI) systems, which include sharp interfacial density gradients on strongly stratified background states, acoustic wave generation and removal at computational boundaries, and stratification dependent vorticity production [1]. The computational framework is used to simulate two-dimensional single-mode RTI to extreme late-times for a wide range of flow compressibility and variable density effects. The results show that flow compressibility acts to reduce the growth of RTI for low Atwood numbers, as predicted from linear stability analysis.

\section{Background and Motivation}

An accurate representation of the stratification, acoustic, and intrinsic compressibility effects involved in the compressible RTI system imposes strong requirements on the numerical scheme, such as resolving the acoustic time scale, handling acoustic waves and RT shock waves at the boundaries, and performing computations over a vast range of density scales. In order to capture the late-time behavior, the simulations need to be performed in long vertical domains. If the background stratification is strong, the density range can span orders of magnitude. In addition, vortices are generated continuously within the RT mixing layer and nonlinearly interact with one another to affect the late-time large-scale growth.

\section{Description}

To minimize the computational effort required for high resolution DNS of compressible RTI, a wavelet-based adaptive mesh is utilized so that the resolution of the computational grid matches the local scale of the system. The wavelets allow the grid to dynamically adapt to the physical features in the flow as they evolve in time while maintaining a direct control of the error. A consistent initialization is developed and implemented for both the thermodynamic variables and the velocity field, such that the generation of acoustic waves is minimized and does not affect the instability growth. In order to isolate the RTI growth from the effects of wave reflections at the boundaries, two different boundary methodologies are also developed and implemented: a diffusion buffer zone that damps acoustic disturbances, and a characteristics-based non-reflecting boundary that removes the energy of acoustic waves from the stratified background. The numerical methodology also includes a dynamic time integration method that can be efficiently applied to highly nonlinear and potentially stiff convective-diffusive systems, such as compressible RTI.

The comprehensive computational framework is applied to simulations of two-dimensional single-mode RTI, which act as a good test for the numerical algorithm by exposing any directional bias. However, the numerical methods are all easily extendable to three-dimensional domains with a multi-mode initialization. Specifically, the boundary conditions are general due to the mostly planar nature of the acoustic waves generated from the growth of compressible RTI. The validation of the methodology includes a resolution convergence study, simulations for a wide range of flow compressibility and variable density effects that remain symmetric well into late times (as seen in Fig. 1), and a look at the flow compressibility effects for the small Atwood number (A) case. 


\section{Comprehensive numerical methodology for direct numerical simulations of compressible Rayleigh-Taylor instability}
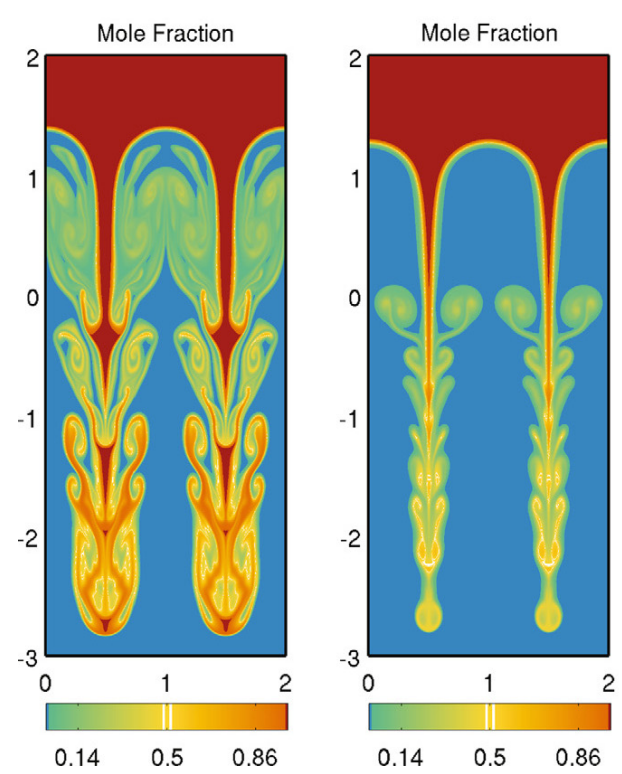

Mole fraction during late-time RTI growth for low-compressibility effects (left) and highcompressibility effects (right) at $A=0.3$.

\section{Anticipated Impact}

The advanced computational framework developed specifically for DNS of compressible Rayleigh-Taylor systems is likely to shed new light on the nature of the RTI. The compressibility effects cannot be summarized by any one single quantity, nor are the effects universal. Stratification is closely tied to the intrinsic compressibility within the system when the initial conditions are in thermal equilibrium, but it is also affected by the molar mass difference between the two fluids. The two effects can be completely decoupled for thermal out-of-equilibrium initial conditions. When the molar mass difference is small, the background stratification acts to suppress the instability growth, and global stability can be achieved quickly. However, when there is a large difference in molar mass, unstable configurations within a highly compressive system may lead to an enhanced growth when compared to the incompressible system. Such results may have important applications to understanding the mixing in the inertial confinement fusion context. The high-resolution simulations needed to address these issues are not possible on today's computers, unless specialized computational strategies are used, such as those developed and implemented for this work.

\section{Acknowledgements}

This work was made possible by funding from the LDRD program at Los Alamos National Laboratory through project numbers 20070195ER and 20090058DR. OVV and SJR were supported by Los Alamos National Laboratory, under subcontract No 57206-001-07. This support is gratefully acknowledged. Computational resources were provided by the LANL Institutional Computing (IC) Program.

\section{References}

[1] S. J. Reckinger, D. Livescu, and O.V. Vasilyev. Comprehensive numerical methodology for direct numerical simulations of compressible Rayleigh-Taylor instability. J. Comput. Phys., 313:181-208, 2016. 